\title{
The impact of culture on AIDS nurses in Iran
}

\author{
Hoda Fotovvat, Mohammad Zahedi Asl \\ Tabatabayi University, Dehkadeh-ye-Olympic, Tehrān, Iran \\ Email: $\underline{\text { Hoda.Fotovvat@gmail.com, Zahediasl@atu.ac.ir }}$ \\ Received 24 September 2013; revised 22 November 2013; accepted 18 December 2013
}

Copyright (C) 2013 Hoda Fotovvat, Mohammad Zahedi Asl. This is an open access article distributed under the Creative Commons Attribution License, which permits unrestricted use, distribution, and reproduction in any medium, provided the original work is properly cited.

\begin{abstract}
AIDS is one of the medical professional's or young person ventures in third world countries like Iran. The growth of AIDS victims in the recent years has made a lot of global worries about cure process and other related issues to HIV problems in these countries. One of the effective groups in the study of AIDS patients is nurses who are in direct contact with patients. Our investigation led to study two variables “consciousness" and "vision" of nurses working in Imam Khomeini hospital of Tehran University of Medical Sciences. Based on the results we achieved that nurses have a low medium degree in the variables studied which is as a result of poor and low effective trainings. In addition to this as a consequence of wrong culture in Iran about AIDS patients or even people in contact with them, nurses have been in a great pressure as they might be in danger of disease transmission. This negative trend has caused a great anxiety about the real condition of AID patients in Iran and their cure process.
\end{abstract}

Keywords: AIDS; Nurses; Culture; Iran; Patients

\section{INTRODUCTION}

AIDS or acquired immunodeficiency syndrome is a new virus spread all over the world and one of the medical professionals or young persons ventures. This disease is rooted in intravenous drug addiction, unemployment, povertry and prostitution [1].

Statistics show that Iran is in the top rank of drug addicted people in the eastern Mediterranean region and Africa so that in 2007 14\% of AIDS patients are from injection [2]. Based on the statistics reported in UNDP conference each minute in Africa and the middle east one person is added to AID patients in the world, so that in 2007 about 44000 new ones are reported in the region
[3].

AIDS is a killing disease. Unfortunately the cure or vaccination for prevention of this is not yet invented. Center of disease control in 1981 has reported that AIDS makes immune system not be able to fight for a long time against infections and malignancies life threats [4]. All around the world AIDS is recognized as the main reason of death in infectious diseases and also the fourth cause of all kinds of death over the world [5]. Based on prescriptions up to 2020 about 200 million people of all world people will be infected with this killer disease.

The first AIDS case in Iran, 6-year-old child hemophilia, was reported in 1987; the disease was caused by a blood transfusion (Integrated Regional Information Networks). From that point up to now 27 years passed. Based on the current statistics from medical universities up to April 2013, 26,565 persons are recognized in Iran [6]. Of course based on statistics of studies of the World Health Organization this estimation should be about 80,000 , as some of suffering people do not introduce themselves to official systems as a result of culture and hallmarks, so the reported people are about 4 times more than official or governmental statistics. In this situation the responsible persons as a consequence of wrong reports of infected persons do not take the situation as serious as they have to, and even believe that their performance is being good as the results show. However the growth of this disease in other segments of society has affected the other parts of society. These segments include people in contact with the patient like acquaintances. The most important people who are in contact after relatives are nurses in the cure process. The special situations of this kind of patients have led nurses to be in a special situation too.

Almost 3 million persons working in the Health Care section encounter annually with different kinds of viruses infused with blood. Over $90 \%$ of infections occurred in these personnel are in countries with low income and most of them are preventable [7]. Although HIV stability 
outside of the body is weak, there is a high probability to infect nurses, doctors, dentists, health workers, service workers and other hospital staff [8]. According to this potential risk and this fact that those patients with HIV have no symptoms for a long time, then there is a high venture for nurses, as nurses are in direct contact with blood and other patient's body fluids, and they most of the time do injection jobs without considering security procedures. According to these risks nurses get nervous in order to do their career jobs for HIV patients. Having enough information about HIV is an important tool to lower their temper pressure [9].

Wrong data about AIDS might affect nurse's vision and then patient's health care qualification decreases. As the general viewpoint about people encountering patients with HIV is about the risks of infection by many ways known or unknown, nurses prefer not to be in conditions possible to be at risk. As AIDS patients show signs of anxiety and withdrawal, nurses responsible for these people might show their preparedness by their visions about AIDS in general [10]. Since there is no vaccine for this disease and also there is no cure, the only way to be protected is to use prevention methods and the best way is to learn them especially to those who need. The first step in this process is to distribute information about different subjects related to this disease, and then make vision and beliefs to change mind paradigms of people related somehow [11]. When prevention programs start, the investigation of increase or decrease of consciousness, vision and behaviors during the program in the society makes essential and this test can show how successful or not the used methods are. So measurement of vision and consciousness and behavior of in danger groups occasionally becomes mandatory.

It is surely known that if we do not have enough knowledge of consciousness and beliefs of people, we might not program efficiently for them [12]. Previous researches show that consciousness condition in $[10,13]$ studies is satisfactory and in some other researches [11-13], is not satisfactory. Also previous researches show that vision condition in $[10,14]$ is not satisfactory and in [15-18] is satisfactory.

Nurses being in direct contact with patients, consciousness improvement and positive vision will make them think positively, and as a result, this mind paradigm will prohibit consequences occurred of bad treatment with patients. The aim of this study is to identify consciousness and vision level of nurses working in health care centers, so that we could use the results to study the HIV patients from different sides. Another goal of this study is to discuss education quality in Iran universities programmed for nurses in AIDS treatment viewpoint.

\section{MATERIALS AND METHOD}

This study is a descriptive - analytical study and is done cross-sectional. Employees of Tehran University of Medical Sciences in 1392 were considered. Samples were selected by random. This selection was based on a list available and under the supervisors' presence. 378 persons were selected. The instrument of research was a questionnaire based on $[19,20]$ works. To test validity we used medical experts of medical science university. We also tested reliability of the questionnaire with a protest by 45 of random samples with 0.87 .

The questionnaire consisted of two parts: the first part of demographic sample, age, gender, years of education, marital status, source of information and the second part was about consciousness and vision. It consisted of 45 questions in general, in which 15 questions were about consciousness and 30 was about vision. Consciousness questions were constructed by yes or no and right ones were marked 1 and wrong or I do not know were marked with 0 . Consciousness question were orderly classified into three sections including weak $(0-15)$, average (15.1 - 22) and strong (22.1 - 30).

Domain of specific knowledge about the nature of the consciousness questions was is in 4 categories including disease transmission, prevention, diagnostic tests, and specific questions about the nature of the disease. Specific Questions of consciousness were in four categories including 5 questions: Diagnostic HIV-positive individuals with symptoms, possible transmission of the disease without symptoms, autoclave killing effect on HIV, HIV-positive individuals with a long life opportunity and the treatment of opportunistic infections, and were categorized into three levels of less than 2.5 poor, 2.15 to 3.75 above the average and 3.75 were classified as good.

Specific knowledge about the nature of the 4 categories of disease transmission, prevention and diagnostic tests were developed. Specific questions about the nature of the disease, including 5 questions: Diagnostic HIVpositive individuals with symptoms, possible transmission of the disease without symptoms, autoclave killing effect on HIV, HIV-positive individuals with possible life and the treatment of opportunistic infections three levels of less than 5.2 poor, 51.2 to 75.3 above the average and 75.3 were classified as good.

To transfer HIV disease consists of 13 questions: Using common bathtub, insect bites, using the toilet, transferring from mother to fetus infected during pregnancy, breastfeeding mothers with newborn infants, splashing fluid in the eyes, sweating the body, using common dining tools, the use of non sterile dental instruments, transmission through intercourse most likely transmitted vaginal (vaginal) than rectal (anal), oral sex transmission, transmission through blood and blood products. In the range of less than $5 / 6.5$ poor, 6.51 to 9.75 in the average level, 9.75 were classified as good.

Diagnostic tests include 4 questions, test result may be negative for an HIV-infected person, windows period can 
be recognized with the contribution of HIV test, having a written policy of the test HIV/AIDS in a test of the most frequently used hospitals, and a simple test confirms HIV. The range was poor for less than 2, 2.13 on average, and higher than 3 were classified as good. Prevention includes 8 questions: prevention by adherence to ethical principles, prevention of sexual transmission of disease by condoms, prevention of sexual transmission of disease by oral contraceptives, prevention of sexual transmission of disease by the cap cervix, preventing of disease transmission by control of transfusion of blood donations, preventing of disease transmission by non-sterile equipment, preventing of disease transmission by drug addiction and common syringe, and by executing prophylaxis in face of blood products. The results were poor in the range 4, 14.1 to 6 were classified as average, and above 6 were classified good.

The items with Likert scale questions related to attitudes to agree, disagree, no opinion was designed. A score of 2 for a agree response was calculated, the response to neutral (neutral) was a score of 1 and a score of zero was given to an unfavorable response. In general, each individual score was a domain of $0-30$ and was ordered in negative (unfavorable), neutral and positive (favorable). Scores below 15 was poor, 22 - 16 average, higher than 22 was considered good. These questions included the willingness to treat HIV-positive patients; HIV-positive patients should be isolated from other patients, and other questions were about the views of nurses about patients, patient confidentiality, and mandatory testing.

Questions were developed by the researcher, so the content validity by experts and scholars of medicine, nursing and epidemiology were evaluated to assess the reliability and internal consistency of the questionnaire, which resulted to an index alpha, $0.76=\alpha$ for vision and a $0.74=\alpha$ for consciousness and for both of them $0.71=$ $\alpha$, respectively. To perform the study, data collectors and other participants were introduced to the project in a meeting which the goals and methods of the study was described. A training course for them to gather data at the end of scission was held too.

Before completing the questionnaire, employees were accounted for the study design and objectives. Filling out forms or entry into the program was completely voluntary. The staffs were assured that their information will be remained confidential. After obtaining verbal consent from them questionnaires were completed by employees without their names \& surnames. The questionnaire was completed within 20 minutes, then the software SPSS were analyzed [21]. Characterizing the data distribution of the tables and analyzing the data by one-way ANOVA, T-test and Spearman correlation coefficients provide a significant level of 0/05.

\section{RESULTS}

Participants were 378 persons. Their mean age was 28 years (minimum 21 years and maximum 33 years). $91.7 \%$ female and the rest male. $28.2 \%$ married, $71.8 \%$ were single.

In this group $29.1 \%$ had good conciseness, $62.9 \%$ moderate and $8 \%$ had poor. The General conciseness mean was $20.3 \% \pm 3.5 \% .47 .5 \%$ of the nurses did not know that the disease is not transmitted through mosquito bites. $33.3 \%$ believed the bathtub transmission of the disease is possible and only $66 \%$ of them knew disease transmission through breast milk. $11.9 \%$ knew that the risk of sexual transmission of rectal (anal) is more than vaginal (vaginal). $44.6 \%$ knew that oral transmission is possible and only $4.5 \%$ of them knew that the most common diagnostic test for HIV is ELISA which can be confirmed by Western blot that in Table 1 have been specified.

A $6 \%$ had a good view, $37.3 \%$ with moderate, and $55.9 \%$ had a poor attitude. Overall, the average level of nurses' attitudes was $14.1 \%$. Table 2 shows the results of the attitude questions. $16.3 \%$ of nurses, answered university education as their source of information about AIDS. $52.8 \%$ of them did not know enough university education about AIDS. The attitude of the staffshad a significant relationship between the information source $(p=0.004)$. But for the attitudes of employees with age, a significant relationship was found. There was a significant relationship between knowledge and attitude $(p=0.001)$ (Table 3).

\section{DISCUSSION}

The greatest spread of HIV infection worldwide, the lack of effective vaccines and treatment, recurrence of a large number of infectious, immunity-devoid diseases resulted from HIV infection, and its threat to the young people and labor forces of Third World societies, including Iran, where people have not still recognized the nature of HIV patients, has become more and more obvious since lack of educational programs caused an unmarked increase in the infection. Furthermore, official authorities consider the statistics on infection reported by their departments sufficient. With no consideration to the statistics released by related organizations worldwide, they refuse to implement measures for decreasing the spread of infection. These facts indicate significance of health care, education, precautionary measures and preliminary steps for controlling the disease effectively.

The findings on medical personnel indicated that awareness of AIDS in the examined group was in moderate-to-appropriate degree. Also, in the study conducted by [10] on the medical personnel in Nigeria, an appropriate degree of awareness was reported. [13] found that 
Table 1. Personality charactristics.

\begin{tabular}{cccc}
\hline Number & Mean of Age & Female $\%$ & Married Females \% \\
\hline 378 & 28 & 91.7 & 28.2 \\
\hline
\end{tabular}

Table 2. Tehran university of medical science employees statistical distribution to HIV disease consciousness evaluation items.

\begin{tabular}{|c|c|c|c|c|c|c|c|c|}
\hline \multicolumn{9}{|c|}{ Questions } \\
\hline \multicolumn{2}{|c|}{ Prevention } & \multicolumn{2}{|c|}{ Experimental Tests } & \multicolumn{2}{|c|}{ Transmission Methods } & \multicolumn{2}{|c|}{ Disease Nature } & \multirow{2}{*}{ Consciousness } \\
\hline Percent & Frequency & Percent & Frequency & Percent & Frequency & Percent & Frequency & \\
\hline 37.2 & 94 & 0.4 & 1 & 104.2 & 106 & 64.5 & 162 & Good \\
\hline 54.2 & 135 & 12.3 & 31 & 47 & 118 & 21.1 & 53 & Medium \\
\hline 8 & 20 & 87.1 & 221 & 10.8 & 37 & 14.3 & 36 & Poor \\
\hline
\end{tabular}

Table 3. Tehran university of medical science employees statistical distribution to HIV disease vision evaluation items.

\begin{tabular}{|c|c|c|c|}
\hline I disagree & $\begin{array}{c}\text { I have no } \\
\text { idea }\end{array}$ & I agree & Items \\
\hline $\mathrm{n}=378$ & $\mathrm{n}=378$ & $\mathrm{n}=378$ & \\
\hline 59 & 12.9 & 28.1 & I prefer not to provide any service for AIDS patients. \\
\hline 4.5 & 3.9 & 91.6 & We can help AIDS patients by giving them consultant. \\
\hline 72.5 & 9 & 18.5 & Social care for patients with HIV positive encourages public to become infected. \\
\hline 21.9 & 7.9 & 70.2 & The AIDS patient must be held in separate parts in the hospital. \\
\hline 25.3 & 10.1 & 64.6 & The AIDS patient must be held in separate hospitals. \\
\hline 55.6 & 13.5 & 30.9 & I can deny giving service to AIDS patient to secure myself and my family. \\
\hline 53.9 & 15.7 & 30.3 & AIDS patients make their family ashamed. \\
\hline 70.8 & 14.6 & 14.6 & Health care budget for AIDS patient is a waste of money. \\
\hline 69.7 & 7.9 & 22.5 & Most of AIDS patients are laissez-faire and deserve this situation. \\
\hline 27 & 7.9 & 64.6 & It is better to do the HIV test intangibly. \\
\hline 30.3 & 5.1 & 64.6 & We must inform the spouse of patients. \\
\hline 12.4 & 4.5 & 83.1 & All nurses working in AIDS section should the tests. \\
\hline 9.6 & 6.2 & 84.3 & HIV patient needs to be marked in order to warn nurses. \\
\hline 41.6 & 15.2 & 43.3 & A nurse with HIV must not work in any section of the hospital. \\
\hline 7.9 & 1.7 & 90.4 & All patients needed to do surgery should test HIV before. \\
\hline
\end{tabular}

most of the studied nurses were aware of HIV. They, however, were not so much cognizant of diagnostic tests. The present study, similarly, showed the awareness of HIV among the respondents was in moderate-to-appropriate degree but the awareness of diagnostic tests was not as much appropriate as it should be. It did not support, however, the findings revealed [7] and [21] reporting an inappropriate degree of awareness respectively among the medical personnel of Australia and of Nigeria. The present paper, also, was not consistent with the findings announced by [22] who indicated the awareness of AIDS was inappropriate among the examined nurses. The inconsistency among the studies is resulted from differences in the selected geographical positions, sources utilized for data collection, and educational methods and from the presence or absence of interests' in seeking new information.
Nurses as the personnel who are in direct connection with infecting causes and AIDS patients must build acute awareness of AIDS in order to conduct effective curable treatment as well as to prevent being infected with HIV. Assessing nurses' awareness of AIDS and of related necessary information indicates they are required to receive further, updated education. The present study demonstrated that most of the respondents were well cognizant of AIDS. However, their awareness of diagnostic tests, modes of sexual transmission and ways of preventing infection resulted from gnat's biting and the toilet was very markedly dawning. As one noticeable cause of infection is sexual transmission, other sources of data collection are required to be regularly utilized in the public since the culture causes the available sources not to develop awareness of improper sexual behavior among people. Special attention to updating nurses' education 
about diagnostic tests, modes of sexual transmission and modes of transmission and non-transmission seems necessary for nurses. The dawning awareness may be resulted from lack of vocational university courses and lack of nurses' own studies. Organizing conferences, seminars and training workshops focusing on the abovediscussed educational requirements could deal with this difficulty.

In the study conducted by [22] on nurses and obstetricians of Babol, the research departments did not take the right attitude towards AIDS. The present study, in which the examined respondents had the wrong attitude, agrees with a number of researches. It, however, is not in line with some other findings. For instance, Simbar et al. (2008) revealed 93.1 percent of the respondents (obstetricians selected from Isfahan medical centers) had a positive attitude. Similarly, the study of Pour sheikhanand Kazemnejad done on laboratory workers of Guilan demonstrated that 91.7 percent took a positive attitude towards health care's in HIV-infected patients. A similar finding was confirmed by 17 Mohebi et al. (2010) who indicated 54.3 percent of personnel of the Shahid Beheshti Hospital and Emam Sajad Hospital in Yasoujtook a positive attitude towards AIDS. Such positive attitude was, also, announced by [18] who carried out their study on nursing students in Germany. The distinctions between these findings and those of the present study are achieved by differences in educational methods, culture, and incentive requirements, existence of safety devices for precautionary measures, existence of apparent law concerning AIDS. Attitudes consist of partly concealed feelings and beliefs about a person's surroundings, being resulted from previous experiences. The assessment of nurses' attitudes displays their tendency towards treatment for AIDS patients. Psychological, social, and economic stresses as well as debility make patients vulnerable. Their requirements are drugs, consulting and medical services, support, and prevention from suspicion, slander and prejudice. As indicated in Table 2, most of respondents preferred the units in hospitals and clinics in which the AIDS patients stay be in quarantine, let alone in the rooms in which they stay. This finding suggests the slander and prejudice against these patients aroused from lack of acute awareness of AIDS. Despite serious attempts at encouraging awareness of the modes of transmission, some misunderstanding still occurs in this regard unfortunately. In the case that efficient, reliable methods are not devised for correcting these misunderstandings and misinterpretations, all attempts at eradicating AIDS epidemic are thwarted. Unjust treatment of medical personnel in AIDS patients causes them to conceal their disease. The concealment and refusal of going to hospitals and medical centers make them not have access to training and consulting services and medical cares. Therefore, they cannot receive urgent information on modes of transmission, precautionary measures and other instructions that they should be given in order to avoid of transmitting the disease to others. And, all crippling effects spread within the society. From the other viewpoint, quarantining HIV-plus patients is regarded scientifically invaluable and rejected. Many of these persons are employed, belong to family and are capable of living under normal conditions. As a result, putting in quarantine causes them to suffer from highly psychological, social, economic and political stresses and contradicts human rights.

Nearly one-third of respondents considered HIV-infected persons disgraceful. Moreover, one-fourth of respondents regarded them indecent persons who should suffer from HIV. Being racked with guilt feeling and being misconduct leads to devastating consequences so far as they may display either pessimism or revengefulness towards themselves or persons probably making them infected. Also, they may consider uninfected persons guilty. As a result, clandestine meetings may be organized by infected persons so far as the AIDS epidemic spreads more easily. As slander and prejudice stem from non-awareness of AIDS, efficient training methods are required to be adopted for nurses through proper scientific sources in order to improve their negative attitudes. Despite most nurses in this study believed all medical personnel and all patients requiring surgery and dialysis should undergo HIV tests, compulsory testing infringes human rights, the most devastating consequence of which is the creating of identity crises. Therefore, they are required to be trained regularly to receive awareness of such consequences.

As indicated in the present paper, nurses considered academic education as the most enormous information source. Therefore, the flow of the related information is expected to be enhanced through further focus on textbooks updated. But, 52.8 percent of nurses considered academic education inadequate. A correlation existed between awareness and attitude, yet the nurses showed negative attitude towards AIDS as the result of variables other than awareness. These variables are required to be examined in relation to the culture of the examined regions.

\section{CONCLUSIONS}

Wrong attitudes and irrationally cultural beliefs and inadequately subjective norms observed among Iran's people for treatment towards AIDS patients cause some difficulty in their care. From the beginning of the first infection of the HIV virus up to now, no reliable statistics on AIDS patients have been released. A large number of the patients are dismissed and their kin has broken their relations with them [23]. They refuse to receive medical 
care because of irrationally cultural beliefs. The unjust treatments and slanders cause the nurses not to give favorable treatments to these patients. Such trend raised worry in world over Iran-resident persons being infected with HIV wished or unwished since the present study indicates an unjust attitude over and inappropriate awareness of these patients among nurses.

Nurses, before others, provide the infected persons with medical cares, yet they often experience fear in this regard. In the case that nurses are beset by worry, they may not demonstrate their overwhelming support. Like other persons of society, nurses have their personal beliefs and attitudes on creating damaging impacts on the medical care. Even if nurses are not aware of slanders against the infected persons or wrong attitudes over providing these patients with medical cares, they are affected by the culture as the result of their profession. Moreover, its damaging impact is felt sooner or later. This vision is true as it is compatible with the Iranian culture characteristics like plurality but not singularity which leads to act mostly based on the majority of the group [24].

Therefore, public training and change of cultural beliefs and values are required to be taken into careful consideration and fundamental changes should be produced by long-term, systematic programs among people. In addition, mass media are required to broadcast about matters related to AIDS. The number of publication and broadcast in this regard is limited so far as the authorities could not execute well-designed programs.

Also, encouraging awareness and adopting the right attitudes of AIDS help the training to extend among nurses, especially before beginning to provide AIDS patients with medical care. The training and development of necessary skills prevent devastating consequences borne by the infected persons. Nurses no longer feel concerned by providing AIDS patients with medical cares and perform their moral and professional duties towards them.

\section{REFERENCES}

[1] Hatami, H., et al. (2009) A comprehensive book on public health. Arjomand Press, Tehran, 2, 947-948.

[2] UNAIDS (2010) Global report UNAIDS report on the global AIDS epidemic. http://www.unaids.org/globalreport

[3] Baijal, P. and Rodney, K. (2009) XVII International AIDS Conference: From Evidence to Action Regional focus. Journal of the International AIDS Society, 12, 34-45. http://dx.doi.org/10.1186/1758-2652-12-S1-S6

[4] Ebong, R.D. (2004) Knowledge and attitudes of nurses toward AIDS: A case study of general hospital Ogoja, Cross river state, Nigeria. Quarterly of community Health Education, 22, 131-144.
[5] Mozafarzadeh, S.H. and Vahdaninia, M. (2008) AIDS literacy among female high school students: A cross sectional study from Iran. Payesh, 7, 173-180.

[6] Fallahi, H., et al. (2013) Living with HIV Consequences. Payesh Journal, 12, 243-252.

[7] Bell, P.F., Williams, A.K., Ross, M.W., Boswarva, P.A., and Strunin, L. (1993) HIV/AIDS: Knowledge and attitudes of accident and emergency healthcare professionals. Association Nurses AIDS Care, 4, 7-14.

[8] Moradi G., Heravi, M. and Parvin M. (2005) Tutorial HIV/AIDS and sexually transmitted diseases, ordered by Ministry of Health and Medical Education. Health Deputy, Center for Disease Control, Publishing Center 22, Tehran.

[9] Dijkstra, A., Kangavaza, E., Martens, C. and Rasker, J.J. (2007) Knowledge about HIV/AIDS and policy knowledge in a South African state hospital. Journal of Social Aspects of HIV/AIDS, 4, 636-639.

[10] UmehCn. Essien, J., Ezedinachi and En. Ross, M.W. (2008) Knowledge, beliefs and attitudes about HIV/AIDS related issues and the sources of knowledge among health care professionals in southern Nigeria. Journal Report Society Health, 128, 233-239.

http://dx.doi.org/10.1177/1466424008092793

[11] Nojoumi, M., Shojaei, H. and Amerian, M.A. (2007) Knowledge of high school students about AIDS Tehran. 6, 41-46.

[12] Imam Hadi, M., Hadian, M. and Jalilvand, M. (2009) Attitude awareness about aids in high school students of Tehran. Journal of Medical Council of IRI, 24, 350-357.

[13] Omisakin, F.D. (2001) Nurses' knowledge and skills about caring for patients with HIV/AIDS in Osun state. Nigeria Journal of Medical, 10, 30-33.

[14] Omidvar, S.H. (2010) Knowledge level and attitude of midwifery and nursing students regarding AIDS. Journal of Babol University of Medical Sciences, 5, 37-41.

[15] Poursheykhian, M. and Kazennajad, E. (2010) Knowledge and attitude of lab staff towards AIDS in Gilan. Feyz, 8, 70-77.

[16] Simbar, M., Shayanmanesh, M., Naheedi, F. and Akbarzadeh, A.R. (2007) Knowledge, attitude and practice of midwives about HIV/AIDS prevention in selected hospital in Isfahan City. Pajoohandeh, 12, 535-540.

[17] Mohebi, Z., Mobaraki, A., AfrasiabiFar, A. and Moshfe, A.A. (2007) Knowledge and attitude of health personnels working in educational treatmental hospitals in Yasuj city regarding AIDS. Armaghan Danesh, 8, 47-54.

[18] Lohrmann, C., Välimäki, M., Suominen, T., Muinonen, U., Dassen, T. and Peate, I. (2000) German nursing students' knowledge of and attitudes to HIV and AIDS: Two decades after the first AIDS cases. Journal of Advanced Nursing, 31, 696-703. http://dx.doi.org/10.1046/j.1365-2648.2000.01326.x

[19] Ries, C., Heisler, M., Amovitz, L., Moreland, R.S., Mafeni, J.O., Anyamele, C., et al. (2005) Discriminatory attitudes and practices by health workers toward patients with HIV/AIDS in Nigeria. Plos Medical, 2, 743-752.

[20] Mazloumi S. and Abbasi, M. (2006) Knowledge and atti- 
tude survey of high school students of Yazd province of Iran about HIV/AIDS. Zahedan Journal of Research in Medical Sciences, 8, 53-63.

[21] Atulomah, N. and Oladepo, O. (2002) Knowledge, perception and practice with regard to occupational risks of HIV/AIDS among nursing and midwifery students in Ibadan, Nigeria. African Journal of Medical Science, 31, 223-7.

[22] Reeder, J.M., Hamblet, J.L., Killen, A.R., King, C.A. and Uruburu, A. (1994) Nurses' knowledge, attitudes about
HIV, AIDS.A replication study. AORN Journal, 59, 450466. http://dx.doi.org/10.1016/S0001-2092(07)70409-X

[23] Li, X., Wang, H., Williams, A. and He, G. (2009) Stigma reported by people living with HIV in South Central China 2009. Journal of the Association of Nurses in AIDS Care (JANAC), 20, 22-30. http://dx.doi.org/10.1016/j.jana.2008.09.007

[24] Mehrabanfar, E. and Nobari, S. (2013) The role of culture in entrepreneurial alertness in iran based on GEM data. Roshd-e-Fanavari Journal, 34, 25-33. 